\title{
Calculation of gravitational waveforms from black hole collisions and disk collapse: Applying perturbation theory to numerical spacetimes
}

\author{
Andrew M. Abrahams, ${ }^{*}$ Stuart L. Shapiro, ${ }^{\dagger}$ and Saul A. Teukolsky ${ }^{\ddagger}$ \\ Center for Radiophysics and Space Research, Cornell University, Ithaca, New York 14853
}

(Received 26 August 1994)

\begin{abstract}
Many simulations of gravitational collapse to black holes become inaccurate before the total emitted gravitational radiation can be determined. The main difficulty is that a significant component of the radiation is still in the near-zone, strong-field region at the time the simulation breaks down. We show how to calculate the emitted waveform by matching the numerical simulation to a perturbation solution when the final state of the system approaches a Schwarzschild black hole. We apply the technique to two scenarios: the head-on collision of two black holes and the collapse of a disk to a black hole. This is the first reasonably accurate calculation of the radiation generated from colliding black holes that form from matter collapse.
\end{abstract}

PACS number(s): 04.70.-s, 04.25.Dm, 04.25.Nx, 04.30.-w

\section{INTRODUCTION}

One of the most significant goals of numerical relativity is to calculate the gravitational waveforms from realistic, astrophysically interesting systems. Until now, obtaining a good estimate of the asymptotic waveform has required long numerical evolutions so that the emitted waves can be propagated far from the source. There, either radiation extraction techniques can be used, or the solution can be matched onto a null-cone integration. Unfortunately, long evolutions are difficult for a number of reasons. These include throat stretching when black holes form, numerical instabilities associated with curvilinear coordinate systems, and the effects of approximate outer boundary conditions. Even if the simulation does not terminate prematurely, these problems usually lead to severe mass nonconservation, which makes the waveforms extracted at late times invalid. Moreover, certain timeslicing conditions and boundary conditions do not allow all collision simulations to progress far enough for the black holes to merge completely; rather they asymptote to a finite separation. (For examples see Ref. [1].) Even when the time slicing allows the black holes to merge, one must integrate long past coalescence in order to extract the complete waveform. At this time, no numerical algorithm is known that deals effectively with all these problems in the context of multidimensional, radiating spacetimes.

Recently, however, an important idea introduced by Price and Pullin [2] provides a possible way to circumvent some of these problems in certain circumstances. Price and Pullin considered Misner initial data repre-

\footnotetext{
${ }^{*}$ Currently at Department of Physics and Astronomy, University of North Carolina, Chapel Hill, NC 27599-3255.

${ }^{\dagger}$ Also Departments of Astronomy and Physics, Cornell University.

${ }^{\ddagger}$ Also Departments of Physics and Astronomy, Cornell University.
}

senting two black holes at a moment of time symmetry. They realized that when the two holes were sufficiently close, the system could be treated as a single perturbed black hole. By applying gauge-invariant perturbation theory, they calculated this perturbation and evolved it using the Zerilli equation. This allowed them to compute asymptotic waveforms and emitted energies. Remarkably, for fairly small separations, the energies and waveforms agreed well with the results of fully relativistic numerical simulations [3]. In a subsequent study, Abrahams and Cook [4] extended this technique and applied it to initial data representing boosted black holes with a common apparent horizon. The radiated energies computed from these initial data sets agreed quite well with those from the fully relativistic simulations for cases in which the initial separations were large.

In this paper, we apply the same technique to numerically evolved spacetimes that at late times can be approximated as a single perturbed black hole. The perturbation of the black hole is computed on a spatial slice from the numerical metric and extrinsic curvature using gauge-invariant perturbation theory. Waveforms are determined by evolving this perturbation to infinity using the Zerilli equation.

The important difference between this method and standard finite-radius radiation extraction techniques (cf. [5]) is the use of the horizon as an inner boundary at which radiation is purely ingoing. Once the horizon forms and the spacetime settles down to a quasistatic state, data from the numerical simulation are no longer required. The simulation can be terminated at a relatively early epoch; it is not necessary to propagate the radiation pulse out to the weak-field regime. Another advantage of the perturbation method is that the effects of backscatter of the waves off the black hole curvature are automatically taken into account via the Zerilli equation integration of the perturbation to large radii. These effects can only be incorporated approximately with standard extraction methods in which an integration over a timelike cylinder is used to separate off near-zone effects. It 
should be emphasized that the perturbation approach requires the formation of a black hole during the numerical simulation. The black hole horizon cuts off the evolution inside the black hole from influencing the waves outside. The method would be inadequate for, say, an oscillating neutron star spacetime, where there is no horizon.

In Sec. II we briefly describe the numerical relativity code and detail the implementation of the gaugeinvariant black hole perturbation method used to compute waveforms. In Sec. III we demonstrate the application of this method to calculations of black hole collisions and disk collapse.

\section{METHODOLOGY}

The numerical spacetimes representing colliding black holes and collapsing disks are generated using a code that solves the field equations of general relativity coupled to a collisionless-particle matter source. Full details of the equations solved and the numerical method can be found in Refs. $[1,6]$. The code employs the maximal time-slicing condition and the quasi-isotropic spatial gauge, and applies to nonrotating spacetimes in axisymmetry. The metric, expressed in spherical polar coordinates, takes the form

$$
d s^{2}=-\alpha^{2} d t^{2}+\phi^{4} e^{2 \eta / 3}\left(d r+\beta^{r} d t\right)^{2}+\phi^{4} e^{2 \eta / 3} r^{2}\left(d \theta+\beta^{\theta} d t\right)^{2}+\phi^{4} e^{-4 \eta / 3} r^{2} \sin ^{2} \theta d \varphi^{2} .
$$

The code solves the Hamiltonian constraint to compute $\phi e^{-\eta / 3}$, solves the momentum constraints for the components $K_{r}^{r}$ and $K_{\phi}^{\phi}$ of the extrinsic curvature, and evolves the variables $\eta$ and $K_{\theta}^{r}$. The kinematical variables $\alpha$ and $\beta^{i}$ are determined from the maximal slicing condition and spatial gauge conditions, respectively. In Ref. [1] the code was used to study head-on collisions and in Ref. [6] it was used to study disk collapse.

A thorough discussion of the perturbed black hole approximation will be published elsewhere [7]. Here we sketch the general idea and list the equations relevant to the current application. The theory of gauge-invariant perturbations of Schwarzschild was first developed by Moncrief [8]. Moncrief showed how to use the ReggeWheeler multipole amplitudes and their radial derivatives to construct unconstrained even- and odd-parity functions that are invariant under infinitesimal coordinate transformations about the Schwarzschild background. These functions satisfy the Zerilli or ReggeWheeler equation for the appropriate $\ell$ mode. For our approach, which involves extraction of the perturbation data on a spatial slice, the background Schwarzschild time derivative of the gauge-invariant function is also required in order to pose initial data for integration of the second-order Zerilli equation (see $[4,7,8]$ ).

From the viewpoint of our approximation, the nonspherical part of the three-metric, the shift, and the extrinsic curvature are treated as perturbations about a static Schwarzschild black hole in the (approximate) exterior region $r \geq M / 2$, where $M$ is the ArnowittDeser-Misner (ADM) mass of the slice. In quasi-isotropic gauge, the static Schwarzschild metric takes the standard isotropic-coordinate form $\left(\beta_{i}=0, \eta=0, \phi=1+M / 2 r\right.$, $\left.K_{i j}=0\right)$. To compute the perturbation using standard gauge-invariant formalism, first we make the background coordinate transformation of the numerically computed metric and extrinsic curvature on a given spatial slice from isotropic to Schwarzschild radial coordinates $r \rightarrow R$, where $R=r(1+M / 2 r)^{2}$. Then we compute the following Regge-Wheeler multipole amplitudes by integrating over coordinate two-spheres at constant $R$ :

$$
\begin{aligned}
& H_{2}^{\ell}=-2 \pi \frac{r^{2}}{R^{2}} \int_{-1}^{1} d x Y_{l 0} \phi^{4} e^{2 \eta / 3} \\
& h_{1}^{\ell}=0 \\
& G^{\ell}=-2 \pi \frac{r^{2}}{R^{2}} \int_{-1}^{1} d x \sqrt{1-x^{2}} \frac{\partial^{2} Y_{\ell 0}}{\partial x^{2}} \phi^{4}\left(e^{2 \eta / 3}-e^{-4 \eta / 3}\right) \\
& K^{\ell}=\frac{\ell(\ell+1)}{2} G^{\ell}-\pi \frac{r^{2}}{R^{2}} \int_{-1}^{1} d x Y_{\ell 0} \phi^{4}\left(e^{2 \eta / 3}+e^{-4 \eta / 3}\right)
\end{aligned}
$$

where $x \equiv \cos \theta$. Note that we have specialized to axisymmetry, and so the azimuthal quantum number $m=0$. We also compute the normal Lie derivatives of the multipole amplitudes:

$$
\begin{aligned}
\mathcal{L}_{\mathbf{n}} H_{2}^{\ell} & =4 \pi \frac{r^{2}}{R^{2}} \int_{-1}^{1} d x Y_{l 0} \phi^{4} e^{2 \eta / 3} K_{r}^{r}, \\
\mathcal{L}_{\mathbf{n}} h_{1}^{\ell} & =2 \pi \int_{-1}^{1} d x \sqrt{1-x^{2}} \frac{\partial Y_{l 0}}{\partial x} \phi^{4} e^{2 \eta / 3} K_{\theta}^{r}, \\
\mathcal{L}_{\mathbf{n}} G^{\ell} & =4 \pi \frac{r^{2}}{R^{2}} \int_{-1}^{1} d x \sqrt{1-x^{2}} \frac{\partial^{2} Y_{\ell 0}}{\partial x^{2}} \phi^{4}\left[-e^{2 \eta / 3}\left(K_{r}^{r}+K_{\phi}^{\phi}\right)-e^{-4 \eta / 3} K_{\phi}^{\phi}\right], \\
\mathcal{L}_{\mathbf{n}} K^{\ell} & =\frac{\ell(\ell+1)}{2} \mathcal{L}_{\mathbf{n}} G^{\ell}+2 \pi \frac{r^{2}}{R^{2}} \int_{-1}^{1} d x Y_{\ell 0} \phi^{4}\left[-e^{2 \eta / 3}\left(K_{r}^{r}+K_{\phi}^{\phi}\right)+e^{-4 \eta / 3} K_{\phi}^{\phi}\right] .
\end{aligned}
$$

From these we form the gauge-invariant function $Q_{\ell}$ [8], 


$$
Q_{\ell}=\left[\frac{2(\ell-1)(\ell+2)}{\ell(\ell+1)}\right]^{1 / 2} \frac{R}{\Lambda}\left(2 N^{2} H_{2}^{\ell}-2 N^{3} \frac{\partial}{\partial R}\left(R K^{\ell} / N\right)+\ell(\ell+1)\left[K^{\ell}+N^{2} R \frac{\partial G^{\ell}}{\partial R}-2 \frac{N^{2} h_{1}^{\ell}}{R}\right]\right)
$$

where $N^{2} \equiv 1-2 M / r$ and $\Lambda=(\ell-1)(\ell+2)+6 M / R$. We also compute its Schwarzschild time derivative $\dot{Q}_{\ell}=N \mathcal{L}_{\mathbf{n}} Q_{\ell}$, where $\mathcal{L}_{\mathbf{n}} Q_{\ell}$ is constructed by replacing the amplitudes appearing in Eq. (10) with their normal Lie derivatives. Note that the normal Lie derivative does not commute with the radial derivative, and so there are additional terms in $\dot{Q}_{\ell}$ involving the radial derivative of $N$. The two functions $Q_{\ell}$ and $\dot{Q}_{\ell}$ provide initial data for integration of the Zerilli equation,

$$
\frac{\partial^{2}}{\partial t^{2}} Q_{\ell}-\frac{\partial^{2}}{\partial r_{*}^{2}} Q_{\ell}+V_{\ell}(R) Q_{\ell}=0
$$

where $r_{*} \equiv R+2 M \ln (R / 2 M-1)$ is the tortoise coordinate and the Zerilli potential is given by

$$
V_{\ell}(R)=N^{2}\left[\frac{1}{\Lambda^{2}}\left(\frac{72 M^{2}}{R^{5}}-\frac{12 M}{R^{3}}(\ell-1)(\ell+2)(1-3 M / R)\right)+\frac{\ell(\ell-1)(\ell+1)(\ell+2)}{R^{2} \Lambda}\right] .
$$

Numerically, the initial data $\left(Q_{\ell}(R), \dot{Q}_{\ell}(R)\right)$ are interpolated onto a fine mesh ranging from $r_{*}=-500$ to $r_{*}=1000$ and the Zerilli equation is integrated using a second-order accurate leapfrog scheme until the perturbation has all been propagated far away from the peak of the potential, $\left|r_{*}\right| \rightarrow \infty$. At large radius the function approaches the even-parity gravitational wave amplitude

$$
r h_{+} / \sin ^{2} \theta=\sqrt{\frac{15}{64 \pi}} Q_{2}(t ; r)
$$

\section{NUMERICAL RESULTS}

Applying the above equations to numerically generated spacetimes is straightforward. However, it is important to have criteria for when we expect the method to give reliable results. Typically, we wait until an apparent horizon has formed and the distortions of this horizon have become fairly small. In the case of collisions, we wait until a common apparent horizon, encompassing both objects, has formed. We quantify the requirement of small distortions by requiring that the proper polar and equatorial circumferences of the apparent horizon be within $10 \%$ of their Schwarzschild values, i.e., $0.9 \lesssim C_{\text {pol }} / 4 \pi M \lesssim 1.1$ and $0.9 \lesssim C_{\text {eq }} / 4 \pi M \lesssim 1.1$. We also require that the area of the apparent horizon be within $5 \%$ of its Schwarzschild value: $0.95 \lesssim \sqrt{A^{2} / 16 \pi M^{2}} \lesssim 1.05$. Our experience is that the first-order perturbation technique becomes inaccurate when the horizon distortion is much larger than the value adopted here.

Consider a concrete example that illustrates our approach. In Fig. 1 we show the radiated energy extracted when using the perturbation method at different times during a numerical simulation. The black hole collision shown, case b1, is discussed below. Figure 2 shows the particle positions and apparent horizon structure at four times during the course of the evolution. The waveform extracted using the perturbation method at each of those times is also shown. After the common apparent horizon forms at a time of about $t / M \simeq 5.0$, the waveform shape and amplitude become fairly constant. The disjoint hori- zons form earlier, at $t / M \simeq 4.0$. The efficiency - the total radiated energy divided by the total mass of the spacetime - is shown in Fig 1 . This also levels off to a nearly constant value once the black holes are encompassed by a common horizon. This effect is to be expected if the calculation is at all meaningful: The final waveforms and energy emission should not depend on when the numerical data are extracted for the perturbation equations once the final black hole has formed and settled down. Eventually, the effects of throat stretching cause the numerical solution to become inaccurate and the radiated energy begins to diverge rapidly. In all cases where the horizon settles down to being nearly spherical while the full numerical solution is still accurate (judged by the

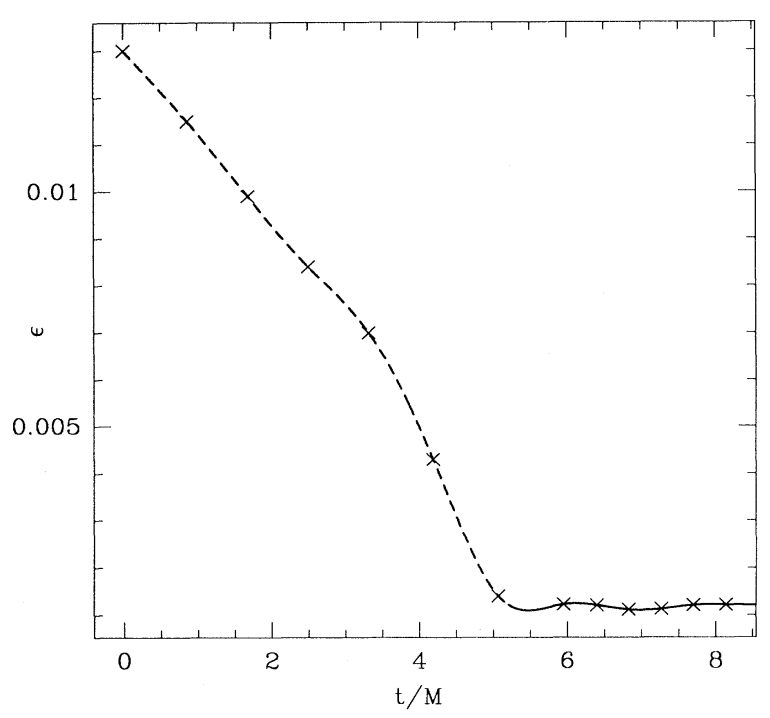

FIG. 1. The radiated energy as a fraction of the total mass energy, determined with the perturbation method, as a function of coordinate time during a simulation of colliding clusters (case b1). The curve changes from dashed to solid at the time an apparent horizon encompasses both clusters. Note that once this common horizon forms, the amount of radiation emitted does not depend on when the perturbation calculation is performed. 

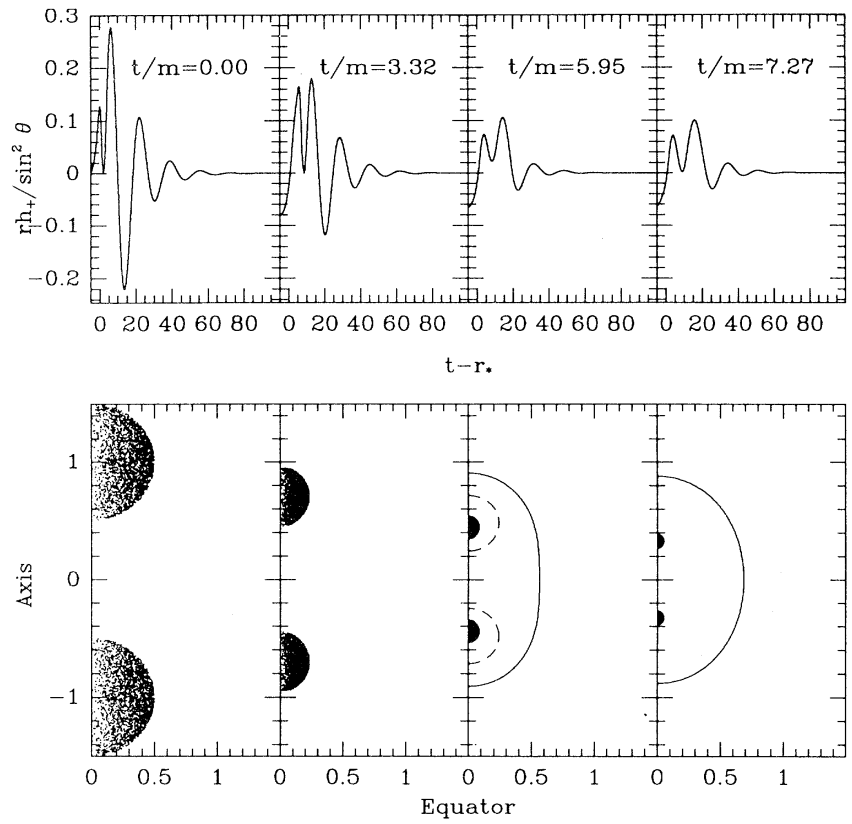

FIG. 2. The perturbation method applied at different times during a numerical simulation. The lower four frames show the particle positions for a collision of initially boosted star clusters (case b1). The dashed lines indicate disjoint marginally outer-trapped surfaces, while the solid line shows the common apparent horizon. The upper four frames (corresponding to the same times) show the asymptotic waveform computed using the perturbation method as a function of retarded time in units of $M$. Radial coordinates are also in units of $M$.

constancy of the Brill mass [1]), the radiated energy remains constant as a function of time as required.

We did encounter some cases where it was impossible to determine radiated energy to better than a factor of 2. Generally this was because the numerical solution became inaccurate before the horizon settled down. These situations are exacerbated by the fact that the quasiisotropic spatial gauge conditions and the Hamiltonian and momentum constraints solved in our algorithm are elliptic equations. Hence, they instantaneously propagate numerical errors to the entire solution, even those occurring inside the black hole horizon. For this reason, even in the relatively accurate cases, we focus on the quadrupole, $\ell=2$ radiation. At present we do not have confidence in the lower-amplitude $\ell=4$ waveforms as the signals are comparable in size to the numerical errors.

Table I lists the 14 cases of colliding clusters and disk collapses that were carefully studied using the perturbation technique. For each case we give the parameters of the initial data including proper separations where applicable. The radiation efficiency is computed for each case. In general, we were able to determine the radiated energy to better than $20 \%$ accuracy, and in many instances to better than $<5 \%$. In the remainder of this section we detail these simulations and discuss the emitted waveforms and radiated energies.

\section{A. Collisions of clusters and black holes}

An alternative to the vacuum, topological black hole approach to simulating black hole collisions is the collision of black holes that form from collapsing matter. Here we consider nonequilibrium spheroids of collisionless matter that separately undergo collapse before hitting each other head on. An advantage of such an approach is that the topology is Euclidean so that simple coordinate systems can be employed. It is also possible to study different scenarios including those in which the clusters of matter do not individually have marginally outer-trapped surfaces surrounding them until after they are both inside a common event (and possibly apparent) horizon. The major disadvantage of this approach is that the matter source must be accurately tracked for the entire simulation, even when it is inside the black hole. This can be very computationally intensive. Of course, horizon boundary conditions that would allow one to excise the black hole interiors from the numerical simulation would solve this problem.

In Sec. II we have briefly described the fully general relativistic collisionless matter evolution code used in these simulations. Full details of this mean-field, particle simulation code, as well as initial data and typical simulations of colliding clusters can be found in Ref. [1]. All simulations discussed here were performed on a 200 radial by 32 angular zone grid with the matter source sampled with 3000 particles. As mentioned above, we monitor the constancy of quasilocal mass indicators such as the Brill mass for a check on accuracy. For the cases shown these resources were sufficient to keep the Brill mass constant within a few percent. This constancy is crucial for the perturbation approach to give consistent results.

First we consider initial data representing two clusters of collisionless matter initially at rest. Since these initial data are conformally flat and at a moment of time symmetry, it is only necessary to specify the cluster radii and separation and solve the Hamiltonian constraint to obtain consistent data. The analytic solution for the conformal factor $\phi$ is given in Ref. [1]. Interestingly, in the vacuum exterior it agrees with the solution given by Misner and Wheeler [9] for two black holes in vacuum. This black hole solution can be thought of as having a threesheeted topology with two Einstein-Rosen bridges, each joining a separate asymptotically flat sheet to a single upper asymptotically flat sheet. For cases in which each cluster initially resides inside its own apparent horizon one would expect identical evolutions to result from the cluster initial data and the three-sheeted data.

Since most numerical studies (cf. Ref. [3]) of two black hole spacetimes have used two-sheeted Misner data where an isometry is imposed between the upper sheet and the single lower sheet, it would be interesting to compare the radiation expected from evolution of two-sheeted initial data with the cluster data to determine the importance of topology. Since both types of data represent the same physical situation - colliding black holes -it would be disturbing if the differences were large. In a future study we will examine this issue in more detail both for timesymmetric and boosted initial data, which is difficult to 
TABLE I. Radiated energy efficiencies from cluster collisions and cold disk collapse.

\begin{tabular}{l|c|c|c|c|c|c|c}
\hline \hline Case & Description & $z_{0} / M^{\mathrm{a}}$ & $R_{0} / M^{\mathrm{b}}$ & $L / M^{\mathrm{c}}$ & $L_{a h} / M^{\mathrm{d}}$ & $v / c^{\mathrm{e}}$ & $\epsilon^{\mathrm{f}}$ \\
\hline c0 & collision & 0.20 & 0.15 & 6.97 & & 0.0 & $1.5 \times 10^{-6}$ \\
c1 & collision & 0.40 & 0.3 & 5.1 & & 0.0 & $1.5 \times 10^{-5}$ \\
c2 & collision & 0.40 & 0.1 & 9.6 & 3.1 & 0.0 & $1.4 \times 10^{-4}$ \\
c3 & collision & 0.475 & 0.3 & 5.7 & & 0.0 & $5.5 \times 10^{-5}$ \\
c4 & collision & 0.50 & 0.1 & 10.2 & 3.6 & 0.0 & $3.0 \times 10^{-4}$ \\
c5 & collision & 0.55 & 0.3 & 6.1 & & 0.0 & $7.5 \times 10^{-5}$ \\
c6 & collision & 0.65 & 0.3 & 6.4 & & 0.0 & $9.0 \times 10^{-5}$ \\
b1 & boosted collision & 1.0 & 0.5 & 6.2 & & 0.05 & $1.2 \times 10^{-3}$ \\
b2 & boosted collision & 1.4 & 0.5 & 6.5 & & 0.15 & $5.0 \times 10^{-3}$ \\
b3 & boosted collision & 0.75 & 0.5 & 5.3 & & 0.05 & $7.0 \times 10^{-4}$ \\
b4 & boosted collision & 0.75 & 0.5 & 5.1 & & 0.10 & $4.0 \times 10^{-3}$ \\
d1 & disk & & 1.00 & & & & $1.5 \times 10^{-4}$ \\
d2 & disk & 1.50 & & & & $3.3 \times 10^{-4}$ \\
d3 & disk & 2.00 & & & & & \\
\hline
\end{tabular}

${ }^{a}$ Coordinate displacement up the axis of the cluster center.

${ }^{b}$ Coordinate radius of cluster or disk.

${ }^{c}$ Proper separation of cluster centers.

${ }^{\mathrm{d}}$ Proper separation of disjoint apparent horizons.

${ }^{\mathbf{e}}$ Boost velocity of cluster as measured by a normal observer.

${ }^{\mathrm{f}}$ The radiation efficiency.

implement numerically in the three-sheeted vacuum picture.

We have studied cluster collisions with a range of separations and compactness. In case $\mathrm{c} 0$ the cluster centers have an initial coordinate separation of only $0.40 \mathrm{M}$ and there is a common apparent horizon on the initial slice. Not surprisingly, this case behaves like a single perturbed black hole. In the top frame of Fig. 3 we show the waveform from this case computed with the perturbation method at an early time during the simulation. The waveform is very reminiscent of that found by Price and Pullin [2] for Misner data in the small separation limit. After a fairly short transient, the waveform is dominated by the lowest order $\ell=2$ quasi-normal-mode oscillations with characteristic wavelength $\lambda=16.8 M$.

For comparison, in the same figure we show the waveform from case $\mathrm{c} 5$ in which the clusters initially have a coordinate separation of $1.1 \mathrm{M}$. Here there are initially no common or disjoint apparent horizons. The common horizon forms at a coordinate time of about $2.0 \mathrm{M}$. The horizon is initially very distorted, $C_{\text {eq }} \simeq 0.62, C_{\text {pol }} \simeq$ 1.16. The perturbation calculation of the waveform and radiated energy is not carried out until $t \simeq 5.3 M$, at which time the horizon has become nearly spherical. Not surprisingly, the wave amplitude is much larger in this case since the initial separation is considerably larger. The waveform shapes are similar but not identical because the cluster infall and collapse are entirely hidden inside the black hole in case c0.

We have also studied initial data, cases $\mathrm{c} 2$ and $\mathrm{c} 4$, for which there are disjoint apparent horizons surrounding each cluster. Since the clusters are more compact, the wave amplitudes in these cases are considerably larger than those from cases with the same coordinate separation but larger cluster radii. For these cases it is possible to compute the initial proper separation of the disjoint apparent horizons. It turns out that the proper separa- tion is indeed larger for these compact cases. For these two cases the radiated energy efficiencies we obtain seem to be compatible with the results from head-on collisions of time-symmetric topological black holes [3]. Unfortunately, the throat stretching is very severe in these cases and it is difficult to have confidence in the energies and waveforms except for cases with very small initial coordinate separations. The simulations lose accuracy (mass error greater than 5\%) within a short time $t \sim 5 M$, and so the perturbation technique is essential for computing waveforms from these cases.

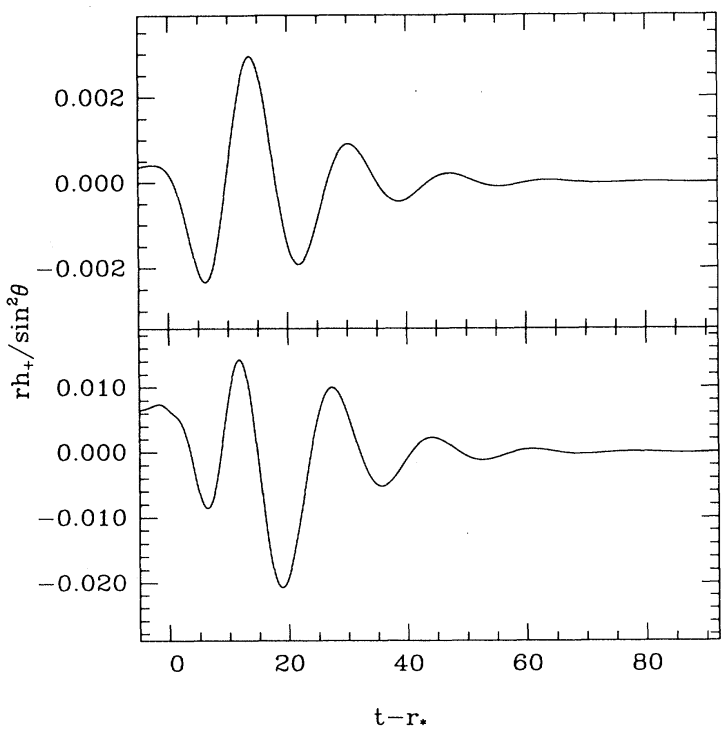

FIG. 3. Waveforms from case c0 (upper frame) and case c5 (lower frame) are compared. The asymptotic gravitational wave amplitude computed with the perturbation method is plotted as a function of retarded time in units of $M$. 
We have also considered cases for which the clusters are boosted towards each other, like those evolved in Ref. [1]. Since these data sets are no longer time-symmetric, it is necessary to solve both the Hamiltonian and momentum constraints for the initial gravitational field. These initial value equations are solved by iteration as described in Ref. [1]. The advantage of the boosted calculations is that the clusters can be started at larger coordinate separations and still collide before the numerical results become unreliable because of the intrinsic collapse of each cluster. As can be seen in Table I, the boosted cases b1-b4 produce considerably more radiation than the unboosted clusters. With boosts, the empirical limit on the radiation efficiency of $\sim 10^{-3}$ for black hole collisions resulting from time-symmetric data (cf. Ref. [4]) can be exceeded. It is not surprising that increasing the boost leads to a more violent collision and thus more radiation. The largest amount of radiation, efficiency $5 \times 10^{-3}$, is produced in case b2, which has the largest separation and boost velocity.

Figure 4 compares waveforms from two boosted clusters, cases b3 and b4. The coordinate separation and initial radii of the clusters are the same in each case; only the boost velocity is different. Clearly, the wave amplitude is much greater in the case of the more violent collision, case b4. The waveforms from the boosted cases all have the same basic qualitative appearance. The initial hump, not present in analysis of boosted Misnerlike data [4], probably arises because the clusters are not initially black holes. Quasinormal modes dominate after about $20 M$ in all cases.

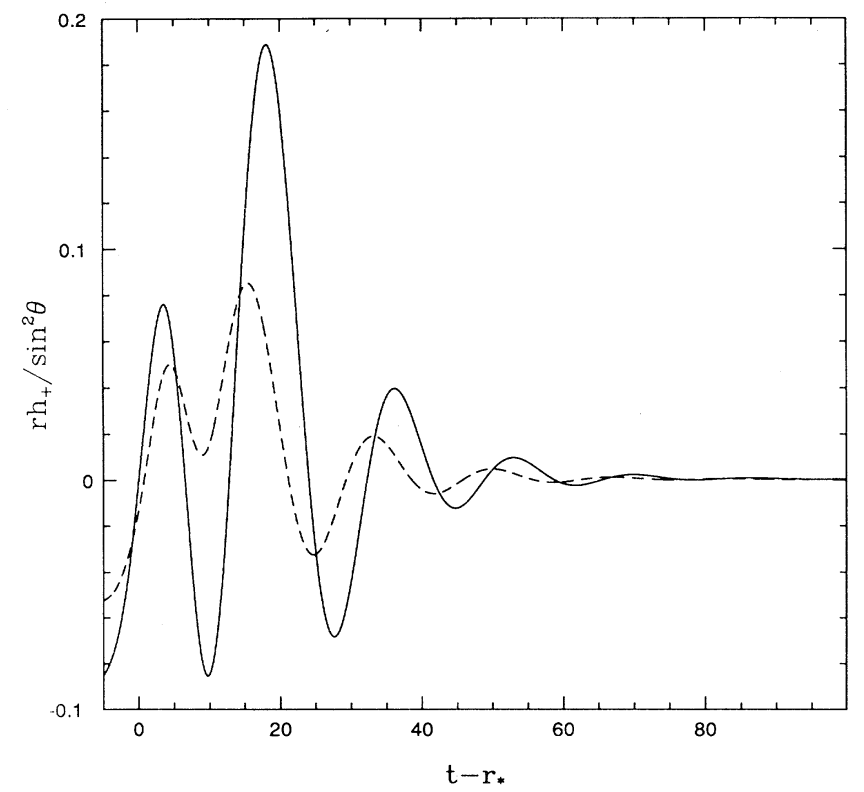

FIG. 4. The gravitational waveform from case b3 (dashed curve) is compared with the waveform from case b4 (solid curve). The asymptotic wave amplitude calculated with the perturbation method is plotted as a function of retarded time in units of $M$.

\section{B. Disk collapse}

Collapsing disks of collisionless matter provide an excellent test problem for axisymmetric numerical relativity codes. Since the source is all in the equatorial plane, the matter evolution equations are all one dimensional. The source is felt by the two-dimensional gravitational field via jump conditions. When the disk matter is initially at rest, the situation provides an interesting analogy to Oppenheimer-Snyder collapse to a black hole in spherical symmetry, but with the added important feature of gravitational radiation production. Since the gravitational field is dynamical in disk collapse, the full machinery of numerical relativity is required to follow the evolution, while spherical Oppenheimer-Snyder collapse is analytic. In Ref. [6] the basic equations for this model were given and a number of test simulations discussed. Here we restrict our attention to cold matter cases that collapse to black holes. By cold matter we mean cases in which the particles in the disk have no velocity dispersion at $t=0$. All simulations shown here were performed on a 300 radial by 16 angular zone grid with the matter source represented by 12000 particles. Even though disk collapse is in a sense the simplest radiating system, in no case are we able to track the evolution long enough to read off the full waveform directly from the simulation. Only with the perturbation method can we extract the entire waveform.

We consider three disk collapses, cases $\mathrm{d} 1-\mathrm{d} 3$ in Table I. As might be expected, the larger the initial radius, the more radiation is produced, although the efficiencies are very small, less than $0.1 \%$ in all cases. Figure 5 shows a typical collapse, case $\mathrm{d} 3$. The matter starts at rest with initial disk radius $R / M=2.0$. The locations of

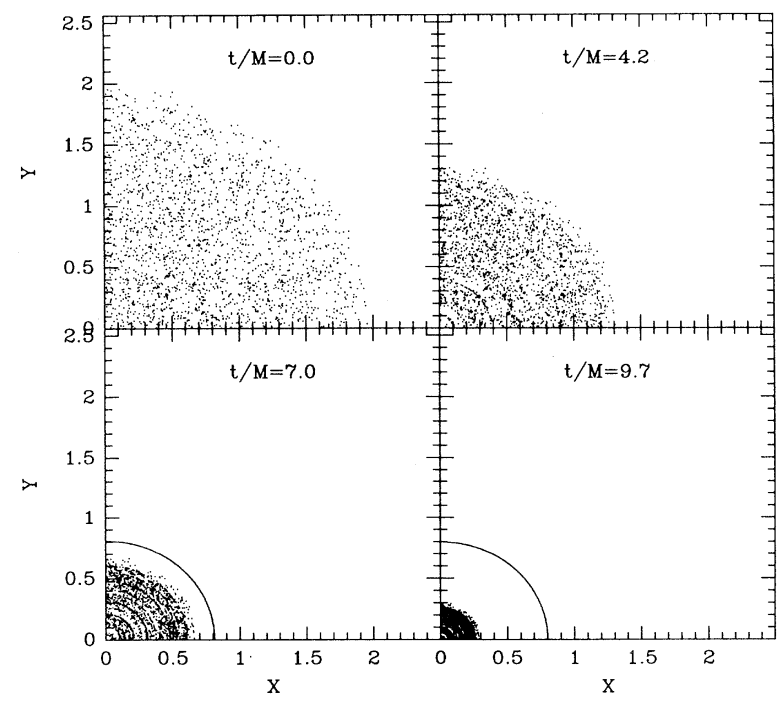

FIG. 5. Particle positions in the equatorial plane are plotted at several times during a disk collapse (case d3). The apparent horizon is indicated by the solid line in the third and fourth frames. 


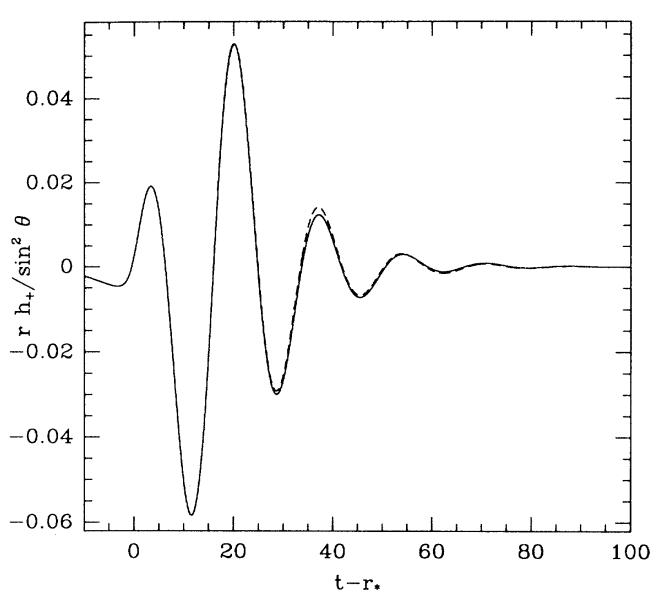

FIG. 6. The gravitational waveform is plotted as a function of retarded time in units of $M$ for disk collapse (case d3) (solid line). The least-squares fit to the two lowest-order $\ell=2$ Schwarzschild quasinormal modes is also shown (dashed line) for $t-r_{*}>17 M$.

the particles in the equatorial plane are shown. After the matter passes inside a radius of about $r / M \simeq 0.5$ an apparent horizon forms. As in Newtonian gravitation, cold disks are unstable to ring formation. As Ref. [1] shows, ring formation here mostly occurs after the black hole forms. The waveform computed using the perturbation approach at a time of about $t / M=8$ is shown in Fig. 6. A least-squares fit to the two lowest-order $\ell=2$ Schwarzschild quasinormal modes is also shown. After an initial transient feature, lasting for about $15 M$, the fit is excellent in both amplitude and frequency.

In Fig. 7 we compare the waveform computed with the perturbation technique (using simulation data at a time of about $t=4.7 M$ ) with that extracted using the usual technique [5] for case d2 (see Ref. [6]). Note that the simulation terminated within about $15 M$ after black hole formation, leaving little time for waves to reach the numerical detector at $r=8 \mathrm{M}$. Only the radiation from the infall phase and the first oscillation of the black hole are obtainable using the standard method before the simulation goes bad. The agreement between the two methods up to this time is reasonable, considering that no special care has been taken in separating out the near-zone field for the infall part of the waveform. This is a delicate matter requiring special determination of initial values for the spacelike extraction techniques [10], but is automatically taken care of in the perturbation approach provided that the waveform is read off the Zerilli integration at a sufficiently large radius.

[1] S. L. Shapiro and S. A. Teukolsky, Phys. Rev. D 45, 2739 (1992).

[2] R. H. Price and J. Pullin, Phys. Rev. Lett. 72, 3297 (1994).

[3] P. Anninos et al., Phys. Rev. Lett. 71, 2851 (1993).

[4] A. M. Abrahams and G. B. Cook, Phys. Rev. D 50, 2364(R) (1994).

[5] A. M. Abrahams and C. R. Evans, Phys. Rev. D 42, 2585 (1990).

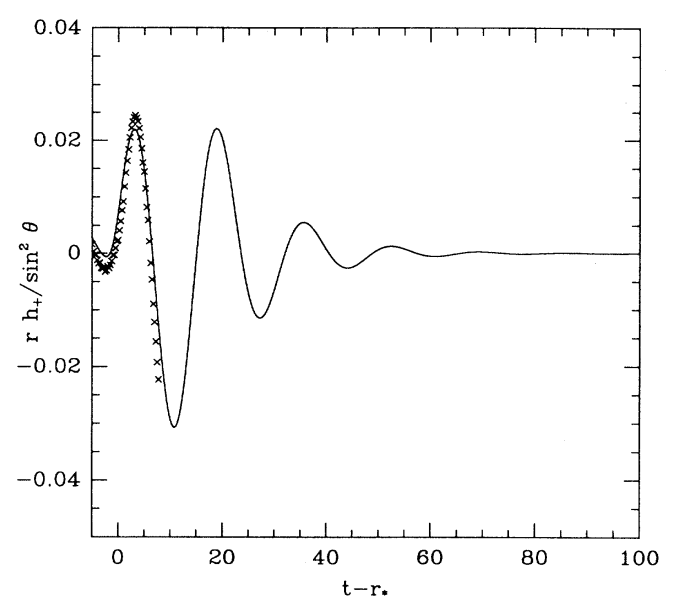

FIG. 7. The gravitational waveform computed using the perturbation method for disk collapse (case d2) is plotted as a function of retarded time in units of $M$. For comparison, the gravitational waveform computed at a radius $r / M=8$ using the standard spacelike radiation extraction technique is also shown (points).

\section{DISCUSSION}

In summary, we have found that the perturbation approach to calculating asymptotic waveforms and radiated energies is effective not only in analyzing initial data sets, but can also be used in conjunction with numerical relativity simulations. For many scenarios it is difficult to evolve black holes long enough to compute accurately the waveforms produced by their formation and ring down. As long as the simulation can be prolonged to the point that a fairly spherical black hole horizon can be identified, the perturbation method presented here should give reliable results. The method holds great promise for more general systems, since it should be possible to extend the method to perturbations of a rotating black hole.

\section{ACKNOWLEDGMENTS}

We thank R. H. Price for stimulating conversations. This work was supported by National Science Foundation Grant Nos. AST 91-19475 and PHY 94-08378 and Grand Challenge Grant No. NSF PHY 93-18152/ASC 93-18152 (ARPA supplemented). Computations were performed at the Cornell Center for Theory and Simulation in Science and Engineering, which is supported in part by the National Science Foundation, IBM Corporation, New York State, and the Cornell Research Institute.

[6] A. M. Abrahams, S. L. Shapiro, and S. A. Teukolsky, Phys. Rev. D 50, 7282 (1994).

[7] A. M. Abrahams and R. H. Price (in preparation).

[8] V. Moncrief, Ann. Phys. (N.Y.) 88, 323 (1974).

[9] C. W. Misner and J. A. Wheeler, Ann. Phys. (N.Y.) 2, 525 (1957).

[10] A. M. Abrahams, in Proceedings of 6th Marcel Grossman Meeting on General Relativity, edited by H. Sato and T. Nakamura (World Scientific, Singapore, 1992), p. 345. 